Instituto Internacional de Investigación y Desarrollo Tecnológico Educativo INDTEC, C.A.

DOI: https://doi.org/10.29394/Scientific.issn.2542-2987.2019.4.12.9.183-200

OAI-PMH: http://www.indteca.com/ojs/index.php/Revista Scientific/oai

Artículo Original / Original Article

\title{
Formación Docente de Educación Inicial en la Atención de Primeros Auxilios
}

Autora: María Eugenia Bolaños Pérez Universidad Pedagógica Experimental Libertador, UPEL mariaeugenia920@gmail.com

Barinas, Venezuela https://orcid.org/0000-0003-0331-6193

Resumen

La ausencia de conocimiento y el efecto emocional que se puede manifestar al momento de encontrarse inmerso en situaciones de riesgo que amerite la aplicación de los primeros auxilios en un momento dado puede generar problemas en las instituciones educativas, en especial en los Centros de Educación inicial. Por ello, el articulo presentado se elaboró con intención de mostrar los avances investigativos, teniendo como propósito general de implementar experiencias formativas en primeros auxilios para los docentes de educación inicial, específicamente en el Centro de Educación Inicial estado Guárico, parroquia Barinas del municipio Barinas estado Barinas, enmarcado en el enfoque socio crítico y el método la investigación acción participante, tomándose como informantes a doce (12) docentes y un (01) coordinador pedagógico del mencionando Centro Educativo, los cuales se seleccionaron porque pertenecen al mismo contexto de estudio. Entre los hallazgos de la investigación destacan un cambio de actitudes de los profesionales educativos hacia la formación, el cual no genera recarga de actividades, sino la utilización de espacios formales y no formales dentro de la institución. Además, se logró la participación activa de los educadores en el desarrollo de las experiencias.

Palabras clave: formación; docencia; bienestar de la infancia. 


\title{
Educational Training of Initial Education in the Attention of First Aid
}

\begin{abstract}
The absence of knowledge and the emotional effect that can be manifested at the time of being immersed in situations of risk that merit the application of first aid at a given time can cause problems in educational institutions, especially in the Early Education Centers. Therefore, the article presented was developed with the intention of showing the research advances, with the general purpose of implementing training experiences in first aid for teachers of initial education, specifically in the Guárico State Initial Education Center, Barinas parish of the Barinas municipality state Barinas, framed in the socio-critical approach and participatory action research method, taking as informants twelve (12) teachers and one (01) pedagogical coordinator of the mentioned Educational Center, which were selected because they belong to the same study context. Among the findings of the research are a change in attitudes of educational professionals towards training, which does not generate recharge of activities, but the use of formal and non-formal spaces within the institution. In addition, the active participation of educators in the development of experiences was achieved.
\end{abstract}

Keywords: training; teaching profession; child welfare. 


\section{Preámbulo}

Los sistemas educativos, en el contexto de la sociedad del conocimiento deben dar respuestas acertadas a las demandas de saberes de los actores sociales, sino brindar una formación integral para enfrentar acontecimientos que pueden suceder tanto en el aula como en los diferentes espacios educativos institucionales. Para ello, el docente asumirá un proceso de actualización, capacitación, profesionalización con temas relacionados a con las disciplinas contenidas en el currículo direccionadas hacia la atención del niño, en especial las referidas a salud integral.

De allí, debe poner en práctica conocimientos sobre la seguridad, higiene como contribuir en la formación de hábitos alimenticios con la finalidad de prevenir, además responder asertivamente ante situaciones de riesgo. En este ámbito de actuación, se inscribe las acciones orientadas hacia la atención en primeros auxilios en los Centros de Educación Inicial, mediante experiencias vivenciales para el fomento de la salud.

Atendiendo a lo planteado, se consideró prioritario la elaboración un artículo con la intención de presentar los avances investigativos propósito general de implementar experiencias formativas en primeros auxilios para los docentes de Educación Inicial, específicamente en el Centro de Educación Inicial Estado Guárico, parroquia Barinas del municipio Barinas estado Barinas, enmarcado en el enfoque el socio crítico y el método la investigación acción participante, considerando los conocimientos previos, así como la viabilidad de las acciones en las instituciones educativas.

Por tanto, al realizar una aproximación ontológica a la formación docente para la atención en primeros auxilios, tomando en cuenta el crecimiento acelerado experimentado en el transcurso del siglo XXI en las ciudades conjuntamente con la población, requiere de un docente formado, capacitado, actualizado y perfeccionado no solamente en su campo disciplinar sino en áreas prioritarias como la salud. Ello obedece, porque en las 
instituciones educativas el infante pasa la mayor parte del tiempo, teniendo posibilidad que en sus espacios ocurran accidentes o situaciones de riesgos que atenten contra su integridad física, emocional y mental, tal como lo plantea Gaintza y Velasco (2017): "los accidentes escolares son una realidad en las escuelas" (pág. 68); los cuales pueden ser atendidos por el personal educativo a través de primeros auxilios o reducirlos con la adopción de acciones sencillas.

Partiendo de esta afirmación, es necesaria en las instituciones educativas venezolanas la formación docente para atender situaciones imprevistas como caídas, golpes, torceduras, picaduras de insectos, entre otros casos a los niños, brindándoles primeros auxilios. Para realizar, esta labor, este profesional debe estar formado en el área, con la finalidad de prestar la ayuda necesaria en emergencias menores mediante cuidados inmediatos como adecuados para atenuar las posibles secuelas y en caso extremo salvar una vida.

En este sentido, los accidentes ocurridos en los Centros de Educación Inicial en el estado Barinas, no deberían ser asumidos por los docentes como hechos aislados ni puntuales, sino como una emergencia que debe ser prevenido mediante la formación y capacitación, previo establecimiento de pautas de comportamientos, además de la ejecución de acciones seguras. No obstante, se evidencia en las referidas instituciones los infantes realizan diversas actividades, así como la práctica deportiva que implican riesgos, debido a ello ocurren accidentes o emergencia que precisan la actuación del docente.

Es importante enfatizar, en estas instituciones los profesionales educativos poseen limitaciones en cuanto a las técnicas de primeros auxilios que pueden ser aplicadas al momento de ocurrir una situación de riesgo, evidenciándose, en ocasiones accidentes como lesiones, desmayos excoriaciones, pinchazo, entre otros. Por tanto, se hace relevante la revisión 
del proceso de formación docente en atención en primeros auxilios en la educación inicial, considerando los conocimientos previos, así como la viabilidad de las acciones en las instituciones educativas.

Por otra parte, la aproximación teórica sobre la formación docente en Educación Inicial ha sido abordada desde diferentes perspectivas, pero prevaleciendo la idea de una actualización como parte de las políticas de Estado, la cual debe realizarse con la finalidad este profesional adquiera, profundice e internalice conocimientos en diferentes disciplinas. Por ello, estas competencias están en congruencia con el compromiso adquiero para la mediación de contenidos a los niños.

En este sentido, Chávez (2004), citado en Arrieta y Silva (2017a), define a la formación docente como:

El proceso mediante el cual, el profesional de educación adquiere las competencias fundamentales en los conocimientos, habilidades y actitudes para ejecutar las acciones docentes, correspondiente a su labor educativa, dando cumplimiento al proceso administrativo, en cuanto a la planificación, organización, ejecución, supervisión y evaluación de actividades, conjuntamente con el desarrollo del liderazgo, comunicación, aplicación de métodos y técnicas, que deberá desarrollar en el ámbito escolar de manera crítica, reflexiva, fundamentada sobre las bases del sustrato ideológico, pertinencia teórico-práctico, así como la idoneidad intelectual (pág. 204).

Lo planteado por las autoras, se direcciona hacia la preparación de este profesional en función de conocimientos proporcionados para el dominio de contenidos del currículo; pero relacionándolos con experiencias de la vida cotidiana de los niños como de los demás actores sociales intervinientes en el Centro de Educación Inicial. Por tanto, su actuación se enmarca más allá de la mediación porque se orienta hacia la transformación del quehacer educativo.

De este modo, el acto formativo adquiere una connotación enmarcada 
dentro de un enfoque técnico-docente, previa consideración de las condiciones profesionales del educador integrándolas en el ser, hacer, aprender y convivir, cumpliendo funciones que requieren la proyección de habilidades, conocimientos como actitudes propias de la enseñanza, haciendo uso de procesos administrativos como la planificación, organización, dirección, ejecución, supervisión, control y evaluación de las actividades.

En consecuencia, esta formación debe verse a juicio de como un proceso Arrieta y Silva (2017b): "como un proceso dinámico, fundamentado en el cambio" (pág. 206); tomando en cuenta los avances científicos y tecnológicos que propician la generación de conocimientos para acercar al docente a la innovación de sus prácticas educativas. En este contexto, reflexiona sobre su actuación pudiendo introducir experiencias significativas que transformen las relaciones de aprendizaje.

Atendiendo a estos señalamientos, la investigadora estima conveniente asumir el modelo de formación relacionado con grupos de investigación y/o acción, en el cual el docente tal como lo señalan González, Zerpa, González y Pirela (2007): "debe desempeñar un papel clave como investigador de su propia práctica" (pág. 281); es decir, no sólo ampliar sus conocimientos sino también mejorar el trabajo en el aula, escuela y comunidad, hasta llegar a transformarlo socioculturalmente, mediante una praxis sustentada en el cambio, la acción como reflexión conjugado con la ampliación de saberes en el ámbito conceptual, reflexivo y práctico.

Es en este nivel de actuación en el cual se inscribe la formación para la atención en primeros auxilios, porque proporciona conocimientos, técnicas y actitudes necesarias en la prevención de situaciones de riesgo físico como emocional en los actores sociales que cohabitan en las instituciones educativas. De acuerdo con Murillo (1998), citado por Alquézar (2010): se entiende por primeros auxilios "los cuidados inmediatos, adecuados y provisionales prestados a personas accidentadas o con enfermedad antes de 
ser atendidos en los centros asistenciales" (pág. 510); implicando, por consiguiente, acciones para conservar la vida, evitar complicaciones y asegurar el traslado hacia instituciones de salud.

Entonces, la formación para la atención en primeros auxilios debe realizarse en los Centros de Educación Inicial para la adquisición de competencias de actuación ante la ocurrencia de accidentes o hechos imprevistos. Para ello, el docente debe responder de una manera rápida como oportuna ante la presencia de situaciones que ameriten el uso de técnicas disponibles en el entorno, activando, principios de acción, orientados hacia revisión del niño para identificar los riesgos encontrados en el lesionado, así como solicitar ayudan inmediata y aplicar procedimientos para evitar complicaciones o la muerte.

Se destaca, esta formación se sustenta desde el punto de vista jurídico en lo establecido en la Constitución de la República Bolivariana de Venezuela (1999), específicamente en el artículo 93:

La salud es un Derecho social fundamental, obligación del Estado, que lo garantizará como parte del derecho a la vida. El Estado promoverá, así como desarrollará políticas orientadas a elevar la calidad de vida, el bienestar colectivo y el acceso a los servicios. Todas las personas tienen derecho a la protección de la salud, el deber de participar activamente en su promoción y defensa, y el de cumplir con las medidas sanitarias y de saneamiento que establezca la ley, de conformidad con los tratados y convenios internacionales suscritos y ratificados por la República (pág. 67).

Derivándose, de este artículo el derecho que poseen los individuos de una protección por parte del Estado de protección a la salud, así como el deber de participar activamente en su promoción, defensa, así como en propuesta direccionadas hacia la ejecución de programas de primeros auxilios sustentados en la protección y participación. Hecho, retomado en la Ley Orgánica de Salud (2006): en el artículo 3, el cual sostiene "el principio de 
participación, que promociona y conserva la salud, mediante la creación de una cultura sanitaria que sirva de fundamento para la prevención tanto a nivel individual, familiar como comunitaria" (pág. 3).

En virtud de ello, la Ley Orgánica de Educación (2009): en correspondencia con el referido marco jurídico, en el artículo 44, señalando la necesidad de brindar orientación al personal educativo para la adquisición de conocimientos mediante actividades de formación, es decir, a través de "una educación extraescolar que atenderá los requerimientos de la educación permanente, a través de programas diseñados que proveerán conocimientos y prácticas para la utilización del tiempo libre" (pág. 39); tendentes a mejorar la calidad profesional del docente, partiendo de la preparación, capacitación, formación en cuento la atención, prevención y cuidado de la salud.

\section{Proyección Metodológica}

Atendiendo a la intención del artículo, la metodología empleada para el desarrollo investigativo, se configuró en el paradigma crítico a partir del conocimiento de la realidad, para mejorar las condiciones del entorno y darle solución a las situaciones problemáticas enfrentadas en las instituciones educativas. De allí, se asuma lo aportado por Melero (2011): "con la finalidad de provocar transformaciones en los contextos en los que interviene" (pág. 343); mediante la reflexión para generar cambios y transformaciones en los actores sociales.

De allí, se asuma como método, la investigación acción participativa como alternativa metodológica, innovadora que involucra a los sujetos en las acciones realizadas para mejorar la situación inicial a través de procesos caracterizados por la acción-reflexión-acción, rompiendo de esta manera, la distancia entre el investigador y el objeto de estudio, que en este caso es la formación docente apara la atención en primeros auxilios en la Educación Inicial. 
En función de éste método, se consideró el desarrollo indagativo a partir de las fases de diagnóstico de la realidad haciendo uso de técnicas e instrumentos de recolección de evidencias, en este caso fue la entrevista a profundidad por medio un guión de entrevista aplicado a tres (3) docentes de educación inicial, planificación de las acciones de formación para la atención en primeros auxilios, ejecución de las acciones mediante la participación activa de los educadores y evaluación con la finalidad de valorar los resultados obtenidos.

De allí, los informantes fueron tres (3) docentes de educación inicial pertenecientes al Centro de Educación Inicial "Estado Guárico", ubicado en la parroquia Barinas del municipio Barinas estado Barinas, los cuales se seleccionaron atendiendo a criterios de inclusión, como los años de servicio, participación y disposición a intervenir en las acciones planificadas. A estos actores se administró la técnica de entrevista a profundidad por intermedio de un guión de entrevista, considerando las categorías apriorísticas: formación docente y atención en primeros auxilios.

Se enfatiza la validez de la investigación, la cual se realizó mediante la triangulación metodológica de la información, existiendo la posibilidad de la investigadora al emplear los mismos métodos llegue a obtener resultados similares. En este sentido, la validez se obtuvo contrastando las respuestas obtenidas en la guía de entrevista aplicada a la docente, en concordancia con las bases teóricas y la percepción de la investigadora.

\section{Diagnóstico}

Para precisar la investigación acción participante fue necesario conocer lo que sucede en la realidad objeto de estudio, es decir, la formación docente para la atención en primeros auxilios en educación inicial; precisando la información aportada por los informantes en los instrumentos de recolección de evidencias, como se destaca a continuación: 
Cuadro 1. Opinión de los docentes respecto a la Categoría Formación Docente.

\begin{tabular}{|c|c|}
\hline \multirow{4}{*}{$\begin{array}{l}\text { Informante } \\
\text { D1 }\end{array}$} & $\begin{array}{l}\text { ¿De qué manera toma en cuenta los conceptos referidos } \\
\text { a primeros auxilios en su práctica pedagógica? }\end{array}$ \\
\hline & $\begin{array}{l}\text { Pocas veces lo hago porque son muy pocos los conceptos } \\
\text { adquiridos sobre este aspecto, aunque me gustaría hacerlo, } \\
\text { pero no poseo el conocimiento necesario. } \\
\text { Me permitiría dar más oportunidades de aprendizaje en el } \\
\text { aula. }\end{array}$ \\
\hline & $\begin{array}{l}\text { ¿Cómo aplica los conocimientos adquiridos sobre } \\
\text { primeros auxilios en los espacios de aprendizaje? }\end{array}$ \\
\hline & $\begin{array}{l}\text { Necesito orientación para poder aplicar los conocimientos en } \\
\text { el aula de una manera efectiva y eficiente. }\end{array}$ \\
\hline \multirow{4}{*}{$\begin{array}{l}\text { Informante } \\
\text { D2 }\end{array}$} & $\begin{array}{l}\text { ¿De qué manera toma en cuenta los conceptos referidos } \\
\text { a primeros auxilios en su práctica pedagógica? }\end{array}$ \\
\hline & $\begin{array}{l}\text { Los conceptos que poseo sobre este tema son limitados, } \\
\text { porque no nos han brindado la oportunidad de asistir } \\
\text { actividades formativas. }\end{array}$ \\
\hline & $\begin{array}{l}\text { ¿Cómo aplica los conocimientos adquiridos sobre } \\
\text { primeros auxilios en los espacios de aprendizaje? }\end{array}$ \\
\hline & $\begin{array}{l}\text { Es difícil aplicarlos, porque son limitados, es decir, no se ha } \\
\text { formado al personal docente en esta área, aun cuando las } \\
\text { orientaciones educativas contemplan la gestión de riesgos. }\end{array}$ \\
\hline \multirow{4}{*}{$\begin{array}{l}\text { Informante } \\
\text { D3 }\end{array}$} & $\begin{array}{l}\text { ¿De qué manera toma en cuenta los conceptos referidos } \\
\text { a primeros auxilios en su práctica pedagógica? }\end{array}$ \\
\hline & $\begin{array}{l}\text { Los conocimientos que poseo son porque me he preocupado } \\
\text { a buscar información, pero es necesario nos brinden } \\
\text { capacitación sobre el tema. }\end{array}$ \\
\hline & $\begin{array}{l}\text { ¿Cómo aplica los conocimientos adquiridos sobre } \\
\text { primeros auxilios en los espacios de aprendizaje? }\end{array}$ \\
\hline & $\begin{array}{l}\text { De una manera muy rudimentaria, porque aún no se ha } \\
\text { recibido formación en aspectos básicos, como por ejemplo } \\
\text { tratar a un niño al cual le pico una abeja. }\end{array}$ \\
\hline
\end{tabular}

Fuente: La Autora (2018); a partir de las entrevistas realizadas.

En el cuadro 1, se aprecia en las opiniones emitidas por los docentes, la ausencia de una formación docente, la cual fue relacionada directamente con la atención en primeros auxilios, evidenciándose, por consiguiente, no se suministran aportes conceptuales como reflexivos necesarios para mejorar la 
acción educativa. En tal sentido, debe brindarse experiencias constructivas a los docentes para la adquisición de conocimientos teórico-prácticos referidos al tema a través de talleres, conversatorios, jornadas, seminarios, entre otros. Respecto a ello, Arrieta y Silva (2017c), señalan:

La formación docente alude al proceso de preparación y actualización del educador a cualquier nivel o modalidad, por lo que es necesario procurar en todo momento formase con las exigencias del sistema y de la sociedad, para de esta manera, alcanzar conocimientos, habilidades y una actitud diferente ante las circunstancias presentes en las instituciones educativas (pág. 217).

Este planteamiento alude al conocimiento como un hecho dinámico, debiendo ser actualizado a medida que se alcanzan innovaciones científicas y tecnológicas para adaptarlo a los requerimientos de las instituciones educativas como de los niños a ser atendidos en los espacios de aprendizaje; además, puede ser ampliado en círculos de formación e investigación para la confrontación de ideas como de las experiencias exitosas. Visto de esta manera, la formación docente para la atención en primeros auxilios en la educación inicial vendría a considerarse una alternativa para el logro de una calidad educativa.

\section{Planificación, Ejecución y Evaluación de la Formación Docente para la Atención en Primeros Auxilios en Educación Inicial.}

A efectos del artículo, la planificación se asumió como un intento para disminuir la incertidumbre, además, establecer previsiones para alcanzar los objetivos deseados, en lugar de limitarse a reaccionar ante los sucesos o las circunstancias que se vayan presentando a la investigadora; apoyándose en los hallazgos develados en la guía de entrevista y la observación efectuada. Asimismo, se destaca la investigación está en proceso, razón por la cual se muestra a continuación la primera actividad efectuada. 
Cuadro 2. Formación Docente (Experiencia 1)

\begin{tabular}{|c|c|c|c|}
\hline \multicolumn{4}{|c|}{$\begin{array}{l}\text { Finalidad: Desarrollar un círculo de formación docente sobre aspectos } \\
\text { conceptuales de la atención en primeros auxilios en educación inicial }\end{array}$} \\
\hline \multicolumn{2}{|c|}{$\begin{array}{l}\text { Contenido: } \\
\text { Primeros auxilios } \\
\text { Estrategia: } \\
\text { Círculo de Formación }\end{array}$} & \multicolumn{2}{|c|}{$\begin{array}{l}\text { Actividades: } \\
\text { Saludo de bienvenida por parte de la investigadora. } \\
\text { Dinámica motivacional. } \\
\text { Orientaciones pedagógicas. } \\
\text { Conformación de equipos de trabajo. } \\
\text { Círculo de lectura sobre primeros auxilios. } \\
\text { Producción escrita por parte de los participantes. } \\
\text { Conclusiones de la jornada. } \\
\text { Evaluación. }\end{array}$} \\
\hline \multicolumn{3}{|c|}{ Recursos Humanos: } & Recursos Materiales: \\
\hline \multicolumn{3}{|c|}{ Investigadora, docentes, directivo } & $\begin{array}{l}\text { Cuadernos, Hojas blancas, } \\
\text { Lápiz, Libro cardenalito. }\end{array}$ \\
\hline Responsable (s): & & ración: & Evaluación: \\
\hline La Investigadora & & oras & $\begin{array}{l}\text { Instrumento: Registro descriptivo. } \\
\text { Técnica: Observación directa. }\end{array}$ \\
\hline \multicolumn{3}{|c|}{ Fecha: 22 de mayo del 2018} & $\begin{array}{l}\text { Indicadores: } \\
\text { Produce textos de manera individual. } \\
\text { Identifica los elementos conceptuales } \\
\text { de los primeros auxilios. } \\
\text { Participación. }\end{array}$ \\
\hline
\end{tabular}

Fuente: La Autora (2018).

Como se muestra en el cuadro 2, el círculo de formación se realizó el día 22 de mayo de 2018 en las instalaciones del Centro de Educación Inicial "Estado Guárico", ubicado en la parroquia Barinas del municipio Barinas estado Barinas, con la participación de los docentes de la referida institución. Asimismo, la docente investigadora dirige un saludo de bienvenida a los asistentes, agradeciéndole a Dios por permitir el emprendimiento de las acciones que servirán para la adquisición de conocimientos sobre la atención en primeros auxilios.

Seguidamente procedió a efectuar la dinámica titulada; el barco se Hunde, se solicitó a los participantes que formaran un círculo; explicándoles: 
El barco se hunde, se hunde, gira a la derecha, gira a la izquierda choca con la roca, el capitán dice: los asistentes deben escribir sus nombres en el aire con una parte de su cuerpo. Durante su desarrollo se observó participación como entusiasmo en los participantes quienes se reían, expresando la utilidad de introducir dinámicas motivacionales en la formación.

Al finalizar la dinámica, la investigadora, les pregunto: ¿Cómo se sintieron?, señalando en voz alta y de forma general: ¡Muy bien! Por su parte, la investigadora considera la motivación implica un impulso hacia un resultado, mientras la satisfacción involucra resultados ya experimentados y los cuales se hacen parte de la actuación.

Posteriormente, la docente investigadora orienta a los participantes sobre los elementos conceptuales de los primeros auxilios, insistiendo en la necesidad de instaurar en las instituciones educativas una cultura preventiva, mediante la adquisición de valores, así como la puesta en práctica de técnicas para auxiliar a los actores educativos en caso de ocurrir accidentes inesperados o situaciones de riesgo.

Luego, se procedió a conformar grupos de trabajos para la realización de lecturas, discusión de lecturas relacionadas con los primeros auxilios, además, de la elaboración de producciones escritas para ser confrontadas por los asistentes. Se realimentó la actividad con preguntas sobre el tema, asimismo, destacó es necesario renovar los escenarios para la formación los cuales deben crear un ambiente rico en posibilidades para que los participantes puedan adquirir información relevante para la construcción del conocimiento.

Evaluación: La docente investigadora realiza la evaluación de las actividades ejecutadas de acuerdo con los indicadores establecidos en el registro descriptivo: indicando la participación activa durante el desarrollo de la estrategia, observándose, la identificación de los elementos conceptuales sobre primeros auxilios, el conocimiento previo. Igualmente, esto accedió que 
se manejaran las dificultades encontradas en el desarrollo obteniendo el logro de la capacidad para manejar el tema tratado, finalmente, se registra las debilidades y fortalezas para ser tomadas en cuenta en las futuras acciones a desarrollar.

\section{Conclusiones}

Dese el punto de vista investigativo, la formación docente para la Atención en Primeros Auxilios en Educación Inicial, se convirtió un espacio para el encuentro entre la investigadora y los docentes. De igual modo, se destaca en el contexto educativo la aplicación de tendencias para la promoción de una concienciación y reconocimiento de la importancia de los primeros auxilios en las instituciones educativas, en especial en los Centros de Educación Inicial, hecho, que reflejará la conformación de ambientes de óptima calidad y de calidad educativa.

Dentro de la variedad de condiciones presentadas que requieren ser incluidas en el sector educativo, es necesario ubicarse específicamente el tema de la formación docente, porque dicho escenario constituye el punto central de la presente investigación. En este sentido, se planificaron círculos de formación docente para la atención en primeros auxilios en el Centro de Educación Inicial "Estado Guárico", ubicado en la parroquia Barinas del municipio Barinas, estado Barinas, encontrándose con los siguientes resultados:

Al aplicarse un instrumento para valorar el desarrollo de cada una de las actividades, se obtuvo en la actividad relaciona con el Circulo de Formación, la motivación alcanzada fue de un 99\% frente al 100\% de participación, $80 \%$ de disposición y un $30 \%$ de conocimiento del tema; pero en la medida que se avanzó estos resultados fueron siendo cambiados tal como lo refleja el gráfico 1, se observa en las mesas, jornadas de trabajo y conversatorio, en las cuales se alcanza a transformar la visión de los temas 
desarrollados.

Gráfico 1. Resultados obtenidos en la valoración de las experiencias formativas ejecutadas

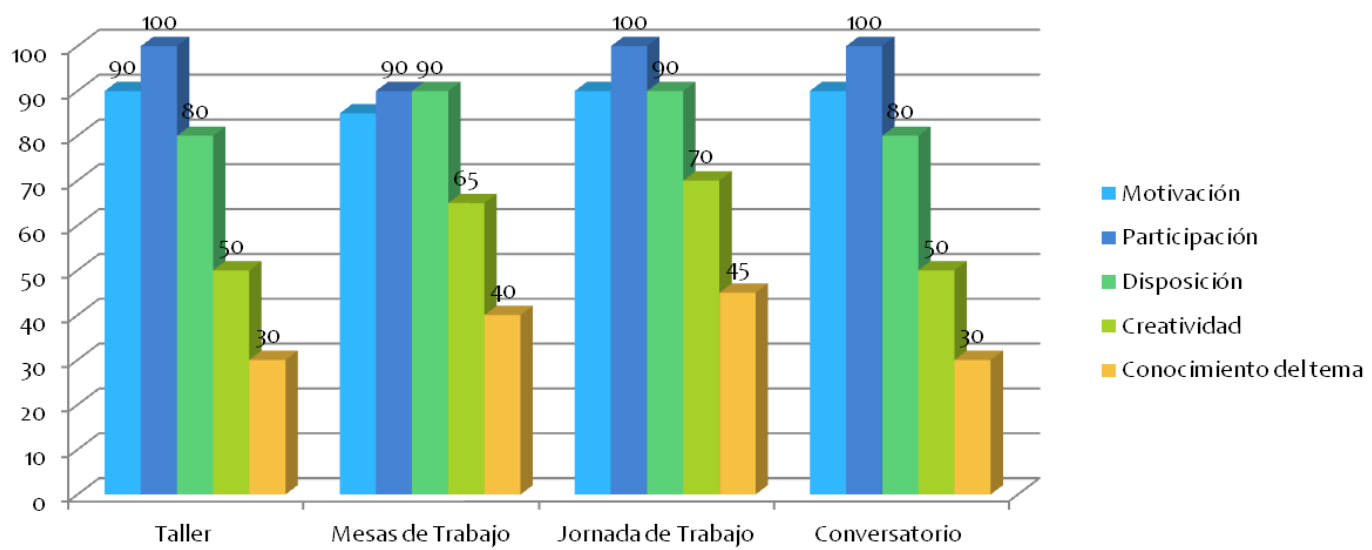

Fuente: Instrumento para la valoración en la ejecución de las experiencias formativas, aplicado por la Autora (2018).

En los resultados del gráfico 1, se reflejan el desarrollo de las experiencias en el tiempo previsto, notándose cambio de actitudes de los docentes hacia la formación docente, el cual no genera recarga de actividades, sino la utilización de espacios formales y no formales dentro de la institución. Además, se logró la participación activa de los educadores porque durante el desarrollo de las experiencias.

En función de ello, se pudo realizar una reconstrucción de las acciones desde el momento inicial que se planteó la investigación y la reunión para la integración del grupo en un equipo de trabajo, los cuales establecieron compromisos, acuerdos, conocimientos previos, las experiencias formativas, así como la identificación de los actores; hecho que conllevo a manejar información para la elaboración de material informativo, así como recursos para el aprendizaje; pero sin perder el objetivo de la investigación, de manera 
que construyeron sinergias mediante nuevas propuestas de acción, para continuar el proceso indagativo.

\section{Referencias}

Alquézar, M. (2010). 41- Primeros auxilios en la educación física: propuesta didáctica para primaria. Revista Arista Digital, (3), 509516. ISSN: 2172-4202. Recuperado de:

http://www.afapna.es/web/aristadigital/archivos revista/2010 diciembr e 41.pdf

Arrieta, L., \& Silva, J. (2017a,b,c). Formación permanente del docente para el desarrollo de estrategias de aprendizaje significativo en el nivel de educación inicial. Impacto Científico, 12(2), 203-220, ISSN: 18365042. Venezuela: Universidad del Zulia.

Constitución de la República Bolivariana de Venezuela (1999). Gaceta Oficial Extraordinaria $\mathbf{N}^{\circ} 36.860$ de fecha 30 de diciembre. Caracas, Venezuela: Asamblea Nacional Constituyente.

Gaintza, Z., \& Velasco, A. (2017). Análisis del grado de formación en primeros auxilios del profesorado activo en educación infantil y primaria. Formación Universitaria, 10(2), 67-77. ISSN: 0718-5006. Recuperado de: http://www.redalyc.org/html/3735/373550473008/

González, N., Zerpa, M., González, D., \& Pirela, C. (2007). La investigación educativa en el hacer docente. Laurus, 13(23), 279-309, ISSN: 1315883X. Recuperado de:

http://www.redalyc.org/articulo.oa?id=76102315

Ley Orgánica de Educación (2009). Gaceta Oficial № 5.929 Extraordinario del 15 de agosto. República Bolivariana de Venezuela: La Asamblea Nacional.

Ley Orgánica de Salud (2006). Ley \# 67. Registro Oficial Suplemento \# 423 de fecha 22 de diciembre. San Francisco de Quito, Ecuador: Sala de 
sesiones del Congreso Nacional del Ecuador.

Melero, N. (2011). El paradigma crítico y los aportes de la investigación acción participativa en la transformación de la realidad social: un análisis desde las ciencias sociales. Cuestiones Pedagógicas, 21, 339-355. Recuperado de:

https://institucional.us.es/revistas/cuestiones/21/art 14.pdf 


\section{María Eugenia Bolaños Pérez \\ e-mail: mariaeugenia920@gmail.com}

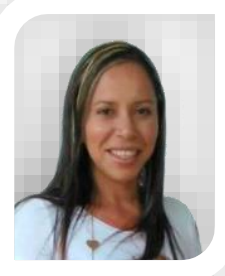

Nacida en la ciudad de Barinas, Venezuela. Inicié en el año 2005 como auxiliar en el preescolar C.E.I., Estado Guárico, Barinas. Me gradué como T.S.U. en Educación Inicial en el año 2009, en el Instituto Universitario de Tecnología "Coronel Agustín Codazzi", donde luego me nivelan como docente de aula, continuo mis estudios en la Universidad Pedagógica Experimental Libertador UPEL-IMPM, extensión Académica Barinas, donde obtengo el título como Profesor de Educación Inicial en el año 2017.

El contenido de este manuscrito se difunde bajo una Licencia de Creative Commons ReconocimientoNoComercial-Compartirlgual 4.0 Internacional 